\title{
Paying in Blood: A Case of Thrombocytopenia in Covid-19
}

\author{
Jahanzeb Malik $^{1}$, Muhammad Javaid ${ }^{1}$, Omaid Majedi ${ }^{1}$, Uzma Ishaq $^{2}$, Tayyaba Zahid ${ }^{1}$ \\ 1. Cardiology, Rawalpindi Institute of Cardiology, Rawalpindi, PAK 2. Hematology and Medical Oncology, Fauji \\ Foundation Hospital, Rawalpindi, PAK
}

Corresponding author: Jahanzeb Malik, heartdoc86@gmail.com

\begin{abstract}
Novel coronavirus disease 2019 (COVID-19) primarily involves the respiratory system. Consumptive coagulopathy is seen in acute respiratory distress syndrome (ARDS) and multi-organ failure (MOF). Usually, only severely sick patients develop mild thrombocytopenia. We present a case of mildly symptomatic young adult, presenting with severe thrombocytopenia. She responded well to corticosteroids.
\end{abstract}

Categories: Infectious Disease, Public Health, Hematology

Keywords: covid 19, thrombocytopenia, covid associated thrombocytopenia

\section{Introduction}

Since its advent in December 2019, the novel Coronavirus Disease 2019 (COVID-19) has infected thousands of people globally with nearly 5500 deaths [1]. COVID-19 primarily produces a respiratory and systemic illness progressing to acute respiratory distress (ARDS) and multi-organ failure (MOF) [2]. It can also involve other organ systems such as hematopoietic and the immune system [3].

Infection with COVID-19 typically presents with fever and upper respiratory symptoms like cough and dyspnea. A small proportion of mildly symptomatic patients present with atypical symptoms like diarrhea, thrombocytopenia, or bleeding tendencies [4]. Commonly, intravascular consumptive coagulopathies are only observed in severely ill patients [5]. A narrow threshold for the identification of COVID-19 should be practiced in the mild disease to prevent delayed diagnosis.

We present a case of COVID-19, diagnosed with an isolated thrombocytopenia.

\section{Case Presentation}

A 29-year-old previously healthy laboratory technician at our institute developed malaise and severe body aches for the past one day. There were no respiratory symptoms, fever, rigors, and chills. She did not report any loss of smell or taste and there were no gastrointestinal symptoms. She gave no history of substance abuse and did not take any medications. There was no history of bleeding or purpura. Her physical examination was unremarkable.

Hematology and biochemistry were ordered. The results showed significant thrombocytopenia. The platelet count was $20 \times 10^{9} / \mathrm{L}$ and hemoglobin was $12.5 \mathrm{~g} / \mathrm{dl}$. There was a relative decrease in lymphocytes (12\%). Her erythrocyte sedimentation rate (ESR) and C-Reactive Protein were $10 \mathrm{~mm} / \mathrm{hr}$ and $6 \mathrm{mg} / \mathrm{L}$ while her D-dimers were negative. Peripheral blood smear showed decreased platelets of $18 \times 10^{9} / \mathrm{L}$ and no schistocytes. Viral serology was negative for HCV-Ab, HIV-Ab, HBsAg and IgM, Herpes, and Cytomegalovirus. Abdominal ultrasound did not show visceromegaly. Chest X-ray and echocardiography were normal. NS-1 for dengue and a thick and a thin smear for malaria was also negative. A hematologist consult was made and a bone marrow biopsy showed increased megakaryocytes (Figures 1, 2, 3). 


\section{Cureus}

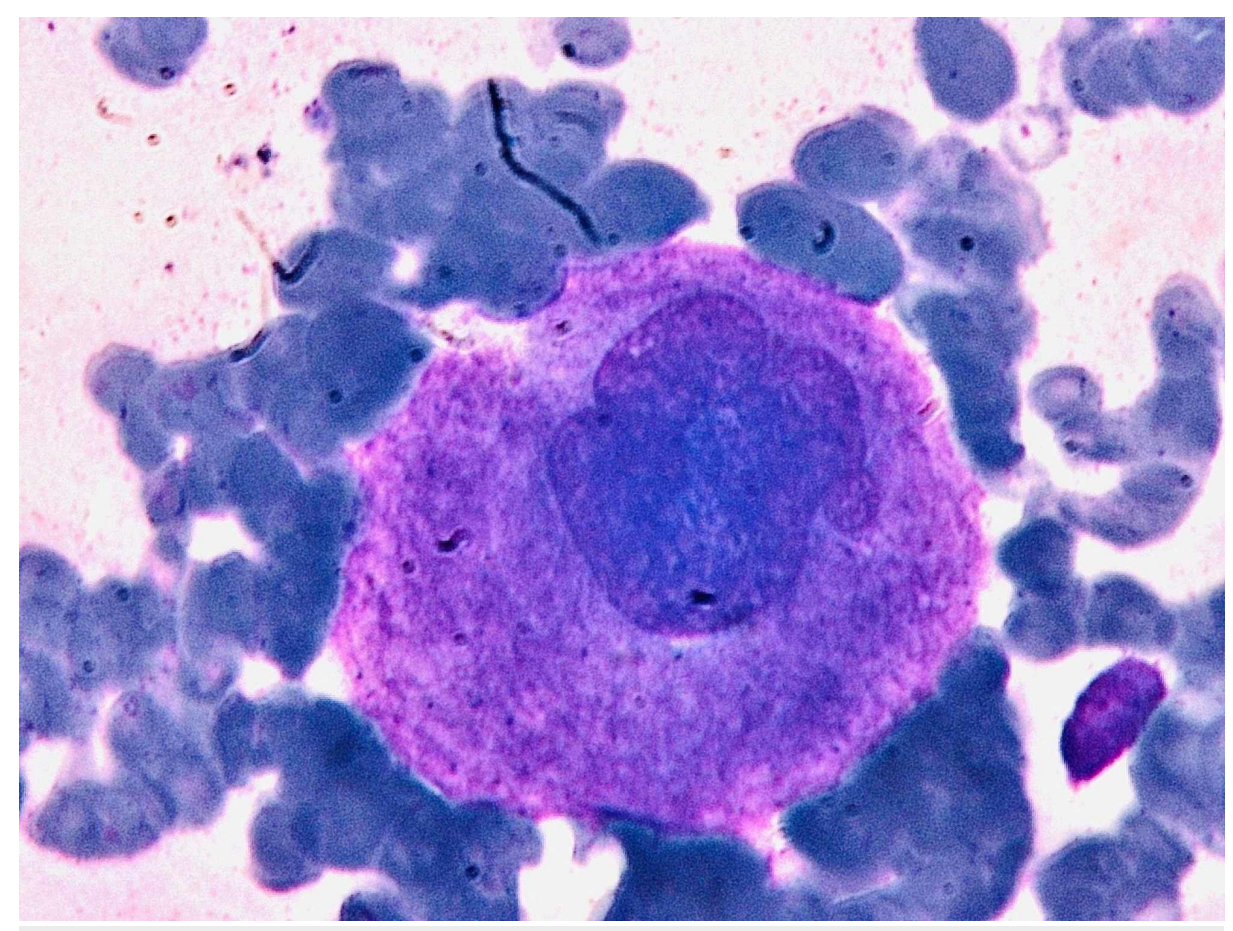

FIGURE 1: Megakaryocyte was identified on bone marrow aspirate at 100x power

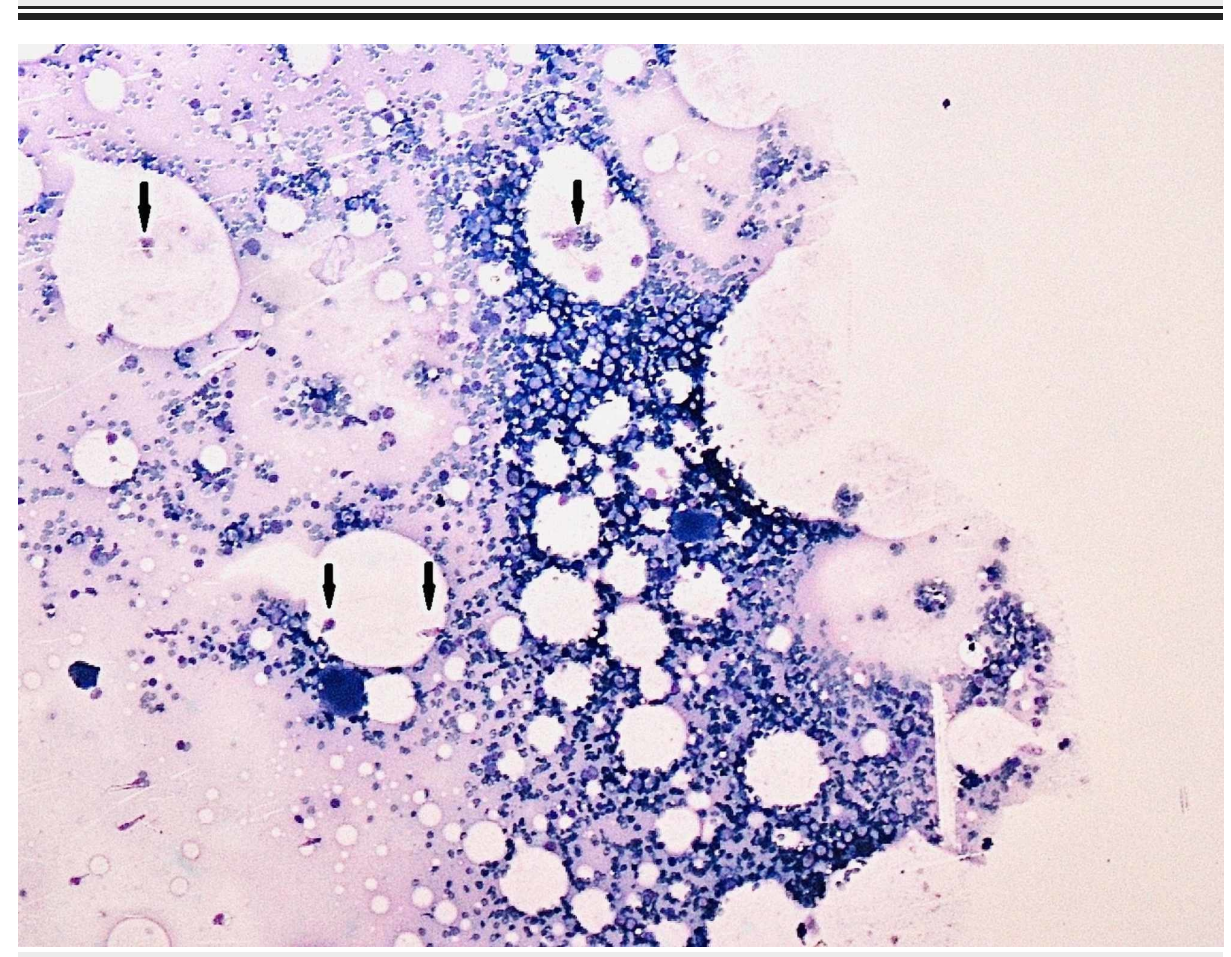

FIGURE 2: Multiple megakaryocytes are seen (Black arrows) on bone marrow aspirate at $10 x$ power 


\section{Cureus}

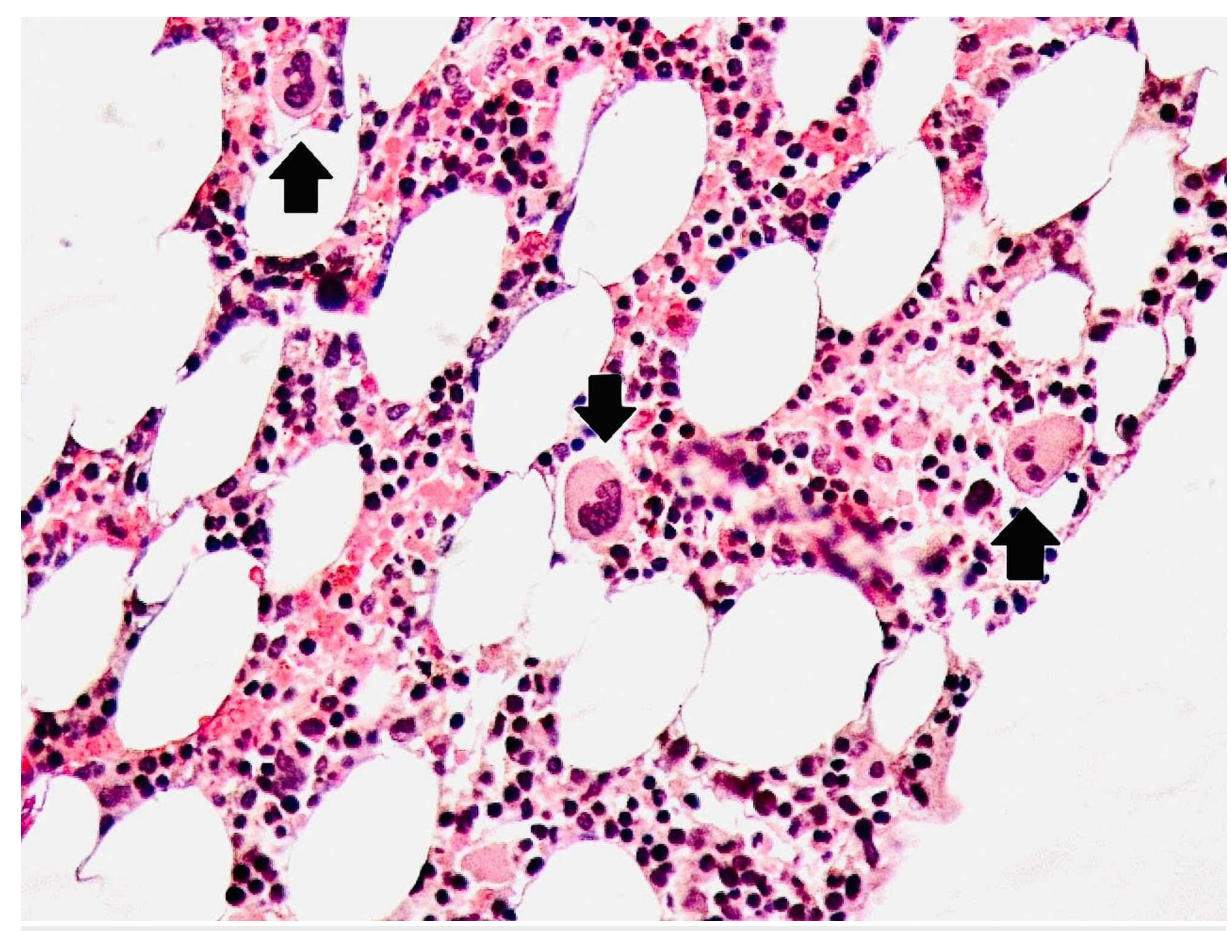

FIGURE 3: Megakaryocytosis confirmed on bone marrow trephine biopsy. This slide shows numerous megakaryocytes (Black arrows).

Considering the looming pandemic, her RT-PCR for COVID-19 was also sent, which came out to be positive. On the following day, her platelets dropped to $7 \times 10^{9} / \mathrm{L}$ and there was myeloid predominance on the peripheral smear. She was transfused four units of platelets. Subsequent platelet counts improved to $12 \times 10^{9} / \mathrm{L}$. She was started on prednisolone $1 \mathrm{mg} / \mathrm{kg}$ in three divided doses.

On the third day, her platelet count was stable at $12 \times 10^{9} / \mathrm{L}$. It increased to $16 \times 10^{9} / \mathrm{L}$ on the fourth day and to $24 \times 10^{9} / \mathrm{L}$ on the fifth day. On the seventh day, prednisolone was stopped as the platelet count reached $47 \times 10^{9} / \mathrm{L}$. Her second RT-PCR for COVID-19 was negative. She was discharged from our institute and on the first follow up after one week, her platelets were $166 \times 10^{9} / \mathrm{L}$. She remained stable on the subsequent followups.

\section{Discussion}

The exact mechanism of hematopoietic abnormalities is not known in COVID-19, but the proposed hypothesis is the increased autoantibodies and immune complexes clear the platelets from the circulation by the immune system. This leads to more platelet destruction and thrombocytopenia [6].

Several studies and case series have described a cascade of consumptive coagulopathies that cause thrombocytopenia [7, 8]. Such studies state that decreasing trend of platelets confer a grave prognosis of COVID-19 infection and lead to a severe disease [8]. One study has postulated that the virus significantly impacts the hematopoietic system leading to pancytopenia [9]. The observation in our patient could be an immunologic reaction commonly seen in viral infections. Surprisingly, all major studies have shown mild thrombocytopenia is a complicated course for the patient [10]. One study demonstrated severe thrombocytopenia with pulmonary and neurological complications.

However, in mildly symptomatic patients, isolated thrombocytopenia is not frequently described. There is only one case report published recently on idiopathic COVID-19 associated thrombocytopenia [11]. Our patient was also mildly symptomatic and after ruling out all major causes of decreased platelets, it was postulated to be associated with the virus.

\section{Conclusions}

Variable presentations can lead to a missed diagnosis of COVID-19. This case report was intended to present a rare case and highlight the proposed mechanism for thrombocytopenia with the immune system working against the hematopoietic cell lines. The role of corticosteroids is evident in this disease and large randomized controlled trials should be conducted for better evidence as a treatment option. 


\section{Additional Information}

Disclosures

Human subjects: Consent was obtained by all participants in this study. Conflicts of interest: In compliance with the ICMJE uniform disclosure form, all authors declare the following: Payment/services info: All authors have declared that no financial support was received from any organization for the submitted work. Financial relationships: All authors have declared that they have no financial relationships at present or within the previous three years with any organizations that might have an interest in the submitted work. Other relationships: All authors have declared that there are no other relationships or activities that could appear to have influenced the submitted work.

\section{References}

1. Li H, Wang S, Zhong F, et al.: Age-dependent risks of incidence and mortality of COVID-19 in Hubei province and other parts of China. Front Med. 2020, 7:190. 10.3389/fmed.2020.00190

2. Geier MR, Geier DA: Respiratory conditions in Coronavirus disease 2019 (COVID- 19): important considerations regarding novel treatment strategies to reduce mortality. Med Hypotheses. 2020, 140:109760. 10.1016/j.mehy.2020.109760

3. Xu P, Zhou Q, Xu J: Mechanism of thrombocytopenia in COVID-19 patients. Ann Hematol. 2020, 99:12051208. 10.1007/s00277-020-04019-0

4. Tay HS, Harwood R: Atypical presentation of COVID-19 in a frail older person . Age Ageing. 2020, 49:523524. 10.1093/ageing/afaa068

5. Lippi G, Plebani M, Henry BM: Thrombocytopenia is associated with severe coronavirus disease 2019 (COVID-19) infections: A meta-analysis. Clin Chim Acta. 2020, 506:145-148. 10.1016/j.cca.2020.03.022

6. Levi M, Thachil J, Iba T, Levy JH: Coagulation abnormalities and thrombosis in patients with COVID-19 . Lancet Haematol. 2020, 7:438-440. 10.1016/S2352-3026(20)30145-9

7. Amgalan A, Othman M: Exploring possible mechanisms for COVID-19 induced thrombocytopenia: unanswered questions. J Thromb Haemost. 2020, 18:1514-1516. 10.1111/jth.14832

8. Tang N, Li D, Wang X, Sun Z: Abnormal coagulation parameters are associated with poor prognosis in patients with novel coronavirus pneumonia. J Thromb Haemost. 2020, 18:844-847. 10.1111/jth.14768

9. Shi W, Lv J, Lin L: Coagulopathy in COVID- 19: focus on vascular thrombotic events [published online ahead of print, 2020 Jul 15]. J Mol Cell Cardiol. 2020, 146:32-40. 10.1016/j.yjmcc.2020.07.003

10. Maquet J, Lafaurie M, Sommet A, et al.: Thrombocytopenia is independently associated with poor outcome in patients hospitalized for COVID-19. Br J Haematol. 2020, Epub ahead of print: 10.1111/bjh.16950

11. Sadr S, SeyedAlinaghi S, Ghiasvand F, et al.: Isolated severe thrombocytopenia in a patient with COVID- 19: a case report. IDCases. 2020, 21:00820. 10.1016/j.idcr.2020.e00820 